\title{
Multi-PRI Signal Processing for the Terminal Doppler Weather Radar. Part I: Clutter Filtering
}

\author{
John Y. N. ChO AND EDward S. ChORnOBOY \\ Lincoln Laboratory, Massachusetts Institute of Technology, Lexington, Massachusetts
}

(Manuscript received 21 September 2004, in final form 22 October 2004)

\begin{abstract}
Multiple pulse repetition interval (multi-PRI) transmission is part of an adaptive signal transmission and processing algorithm being developed to aggressively combat range-velocity ambiguity in weather radars. In the past, operational use of multi-PRI pulse trains has been hampered due to the difficulty in clutter filtering. This paper presents finite impulse response clutter filter designs for multi-PRI signals with excellent magnitude and phase responses. These filters provide strong suppression for use on low-elevation scans and yield low biases of velocity estimates so that accurate velocity dealiasing is possible. Specifically, the filters are designed for use in the Terminal Doppler Weather Radar (TDWR) and are shown to meet base data bias requirements equivalent to the Federal Aviation Administration's specifications for the current TDWR clutter filters. Also an adaptive filter selection algorithm is proposed that bases its decision on clutter power estimated during an initial long-PRI surveillance scan. Simulations show that this adaptive algorithm yields satisfactory biases for reflectivity, velocity, and spectral width. Implementation of such a scheme would enable automatic elimination of anomalous propagation signals and constant adjustment to evolving ground clutter conditions, an improvement over the current TDWR clutter filtering system.
\end{abstract}

\section{Introduction}

Range-velocity (RV) ambiguity is one of the most crucial data quality challenges facing ground-based weather radars. For C-band radars such as the Terminal Doppler Weather Radar (TDWR) ambiguity is more severe as compared to S-band radars such as the Weather Surveillance Radar-1988 Doppler (WSR88D). Thus, to mitigate RV ambiguity for the TDWR, a more aggressive approach must be taken. With diversity in pulse repetition interval (PRI) and pulse transmission phase available as options (frequency and polarization diversity are not), multi-PRI and phase-code techniques can be applied to this problem. For rangefolding protection, these two approaches have complementary strengths and weaknesses (Cho et al. 2003). Multi-PRI signals can be processed to effectively separate different-trip weather even if the overlaid powers are strong or spectrally wide, as long as the overlaid weather does not continuously span a large distance in range. Phase-code processing works well for trip separation, even if the overlaid storm has a long continuous range, but breaks down in cases of strong and/or spectrally wide overlays. Therefore, we proposed an adaptive solution where, for low-elevation tilts, information

Corresponding author address: John Y. N. Cho, Lincoln Laboratory, MIT, S1-659, 244 Wood St., Lexington, MA 02420-9185. E-mail: jync@mit.edu from an initial long-PRI scan would be used to select multi-PRI or phase-code waveform/processing on a radial-by-radial basis in the subsequent scan (Cho 2003).

Although phase-code processing is well established (e.g., Siggia 1983; Sachidananda and Zrnić 1999) the operational application of multi-PRI techniques has been hampered, in large part, by the difficulty of clutter filtering. Because nonuniform sampling aliases power from nonzero Doppler frequencies to the ground clutter band around zero, the clutter filtering also removes power from the aliased frequencies and distorts the phase response at those frequencies. The phase distortion in turn leads to the degradation of velocity estimates. Banjanin and Zrnić (1991) proposed an approach for dual-PRI staggered signals that employs decision logic in choosing between the output of two filter types (split uniform and staggered) to optimize the magnitude response and phase linearity. Good velocity estimates were achieved, however, for only $60 \%$ of the extended unambiguous interval. Sachidananda and Zrnić (2000) devised a technique that deconvolves the effects of nonuniform sampling in the spectral domain, but it only works well if the sample sequence takes a certain form, such as a dual-PRI staggered sequence with a stagger ratio equal to a ratio of small integers. Since we need to use several PRIs to achieve satisfactory range-fold protection, this clutter filter cannot be applied.

A finite impulse response (FIR) clutter filter design 
yielding an excellent balance of magnitude response and phase linearity was introduced by Chornoboy (1993) for block-staggered PRI pulse trains. Filters using this design algorithm are employed in the Weather Systems Processor (WSP) channel of the Airport Surveillance Radar-9 (ASR-9) (Weber 2002) for dual-PRI signals. The same design algorithm can be used for block-staggered signals with more than two PRIs. In this paper we present clutter filters generated using this algorithm for application to a multi-PRI pulse train proposed for the TDWR and examine their characteristics. In a subsequent paper (Part II) we will present the RV ambiguity mitigation techniques to be used on these multi-PRI signals.

A drawback of clutter filtering in the time domain is the difficulty in determining the optimal clutter suppression level for a given range-azimuth cell in real time. Clutter residue maps are sometimes used to select a filter from a range of suppression levels (e.g., Weber 2002). However, ground clutter is not static over time. Vegetation changes with the seasons; construction and demolition alter buildings, roads, bridges, and towers; weather transfers water and ice to and from structures; and winds sway signs and rustle leaves. Anomalous propagation (AP) can also introduce ground clutter to range gates that are usually clutter free. Therefore, ideally, the clutter suppression level should be determined without resorting to a preset clutter residue map. We propose in this paper an algorithm to accomplish this task and test it with simulated data.

\section{Filter design equations}

For a signal with variably spaced samples, a different set of FIR filter coefficients (the $k$ th filter) must be generated for each unique order of sample spacing in a given length of data. If the filtering process for each dwell is to be confined to input samples contained within that dwell, then the number of filters required will equal the number of samples, $N$. The filtering will then be accomplished by multiplying the $N \times N$ coefficient matrix $\mathbf{h}$ (where the $k$ th filter corresponds to the $k$ th row) with the column vector containing the input signal samples. The derivation of the design equations was previously given (Chornoboy 1993), but since it was only released as a conference preprint we shall reiterate it here.

The filter coefficients are produced by minimizing the mean-squared error between the filter response and the desired response in the frequency domain. The frequency response of the filter is given by

$$
H_{k}(\omega)=\sum_{n=0}^{N-1} h_{k n} \exp \left(j \omega \tau_{n}\right)
$$

where $h_{k n}$ are the elements of $\mathbf{h}, \omega$ is the frequency (discretely defined for $M$ points), and $\tau_{n}$ is the time of the $n$th input sample (relative to the beginning of the filter). Then the approximation error is given by

$$
\mathbf{E}_{k}=\mathbf{F} h_{k}^{\mathrm{T}}-\mathbf{D G}_{k},
$$

where $\mathbf{F}$ is an $M \times N$ input transform matrix defined by $F_{m n}=\exp \left(j \omega_{m} \tau_{n}\right), \mathbf{h}_{k}$ is the $k$ th row vector of $\mathbf{h}, \mathbf{D}$ is an $M \times M$ diagonal matrix with elements defining the desired magnitude response in the frequency domain, and $\mathbf{G}_{k}$ is an $M$-length column vector defined by $G_{k m}=$ $\exp \left(j \omega_{m} t_{k}\right)$, where $t_{k}$ is the output sample time. The superscript $\mathrm{T}$ denotes transpose.

Expression (2) gives the error in the complex frequency domain. For radar clutter filtering applications, however, we wish to introduce a separate error minimization for phase. We need low phase errors (high phase error weighting) in the pass band for accurate velocity estimation, whereas in the stop band we need high magnitude error weighting but do not care about the phase errors.

If we let $\varepsilon_{k}(\omega)=\omega t_{k}-\angle H_{k}$ be the phase error for filter $k$, and if $\left|\varepsilon_{k}\right|<\pi / 2$, then by trigonometric inequality we have

$$
\left|\varepsilon_{k}\right| \leq \frac{\pi}{2}\left|\sin \left(\omega t_{k}-\angle H_{k}\right)\right| .
$$

From (1) we get

$$
\sin \angle H_{k}=\frac{1}{\left|H_{k}\right|} \sum_{n=0}^{N-1} h_{k n} \sin \omega \tau_{n}
$$

for $\left|H_{k}\right| \neq 0$, appropriate for the pass band. Combining (3) and (4) we get

$$
\left|\varepsilon_{k}\right| \leq \frac{\pi}{2\left|H_{k}\right|}\left|\sum_{n} h_{k n} \sin \left[\omega\left(t_{k}-\tau_{n}\right)\right]\right| .
$$

Ignoring the $\left|H_{k}\right|$ term, we can write the phase error matrix as

$$
\mathbf{E}_{\mathrm{Ph}, k}=\mathbf{P}_{k} h_{k}^{\mathrm{T}},
$$

where $\mathbf{P}_{k}$ is an $M \times N$ matrix with elements $P_{m n}=$ $\sin \left[\omega_{m}\left(t_{k}-\tau_{n}\right)\right]$. The hybrid, weighted mean-squared error can then be written as

$$
\left\|E_{\mathrm{Hyb}, k}(\omega)\right\|^{2}=\mathbf{E}_{k}^{\dagger} \mathbf{W E}_{k}+\mathbf{E}_{\mathrm{Ph}, k}^{\mathrm{T}} \mathbf{W}_{\mathrm{Ph}} \mathbf{E}_{\mathrm{Ph}, k}+\mathbf{h}_{k} h_{k}^{\mathrm{T}},
$$

where $\mathbf{W}$ and $\mathbf{W}_{\mathrm{Ph}}$ are $M \times M$ diagonal matrices with the magnitude and phase error weights versus frequency as the elements, and the final term is inserted to avoid degeneracy in extreme cases. The superscript $\dagger$ denotes the conjugate transpose. After some matrix manipulation (7) becomes

$$
\begin{aligned}
\left\|E_{\mathrm{Hyb}, k}(\omega)\right\|^{2}= & \mathbf{h}_{k}\left(\mathbf{F}^{\dagger} \mathbf{W} \mathbf{F}+\mathbf{P}_{k}^{\mathrm{T}} \mathbf{W}_{\mathrm{Ph}} \mathbf{P}_{k}+\mathbf{I}\right) h_{k}^{\mathrm{T}} \\
& -2 \mathbf{h}_{k}\left(\mathbf{F}^{\dagger} \mathbf{D} \mathbf{W} \mathbf{G}_{k}\right)+\mathbf{G}_{k}^{\dagger} \mathbf{D} \mathbf{W D G},
\end{aligned}
$$


where $\mathbf{I}$ is an identity matrix. For real filters (8) is a quadratic matrix equation with the minimization solution

$$
h_{k}^{\mathrm{T}}=\left[\operatorname{Re}\left(\mathbf{F}^{\dagger} \mathbf{W} \mathbf{F}\right)+\mathbf{P}_{k}^{\mathrm{T}} \mathbf{W}_{\mathrm{Ph}} \mathbf{P}_{k}+\mathbf{I}^{-1} \operatorname{Re}\left(\mathbf{F}^{\dagger} \mathbf{D} \mathbf{W} \mathbf{G}_{k}\right) .\right.
$$

\section{Filter specifications}

The range of usable PRIs for velocity estimation on the TDWR is limited at the upper end by the coherence criterion, PRI $<\lambda / 4 \pi \sigma_{v}$, where $\lambda$ is the radar wavelength (about $5.3 \mathrm{~cm}$ for TDWRs) and $\sigma_{v}$ is the velocity spectral width. For $\sigma_{v}=4 \mathrm{~m} \mathrm{~s}^{-1}$ [corresponding to median values observed in squall lines; Fang et al. (2004)] this yields PRI $<1050 \mu \mathrm{s}$. For high-elevation scans the shortest PRI is determined by the transmitter capability $(518 \mu \mathrm{s})$, but for low-elevation scans the shortest PRI is set by the Federal Aviation Administration (FAA) operational coverage requirement of $48 \mathrm{n}$ mi in range (594 $\mu \mathrm{s})$. Since the RV ambiguity is worst for the lowest elevation scan, this is the case we will be concerned with in the remainder of the paper.

For range-fold protection of the first trip, which is one of the top data quality issues relevant to the TDWR's mission of weather surveillance in and around the airport, we wish to employ a number of PRIs spread out more-or-less evenly throughout the available PRI range. Since range folding occurs from different range locations for different PRIs, received signals on PRIs that are contaminated by out-of-trip weather, as determined from the initial long-PRI scan, can then be eliminated from further processing. A velocity dealiasing capability should also be available using the remaining, uncontaminated sets of PRI pulses. For this purpose, specific PRI values can be chosen to optimize Chinese remainder theorem dealiasing performance. Further details will be given in Part II of this paper.

We select the following set of PRIs for our clutter filters: 597, 630, 672, 709, 796, 840, 896, and $945 \mu$ s. Note that within this set there are seven pairs of simple integral ratios $(2 \times 2: 3,4 \times 3: 4$, and $1 \times 4: 5)$ that can be employed for velocity dealiasing. Some $n$ consecutive pulses of each PRI can be transmitted per dwell. We call this type of configuration a multiblock-staggered (MBS) PRI sequence. The dwell time determines the maximum $n$ that can be chosen. If a smaller number of PRIs is used, then $n$ can be increased correspondingly. There is an inherent trade-off between range-fold protection possibility (more PRIs) and parameter estimation quality (larger $n$ ). Since these pulse trains are to be selected based on an initial long-PRI scan, we can have more than one multi-PRI sequence available in the menu. In the interest of brevity, we will only present results for a $4 \times 16 \mathrm{MBS}$ sequence $(630 \times 16,709 \times 16$, $840 \times 16,945 \times 16 \mu \mathrm{s})$ in this paper. Three pairs of simple integral ratios $(2 \times 3: 4$ and $1 \times 2: 3)$ are available for velocity dealiasing. This sequence fits within a dwell time of $52.6 \mathrm{~ms}$ currently used by TDWR in the monitor scan mode $\left(1^{\circ}\right.$ azimuth sectors at $19^{\circ} \mathrm{s}^{-1}$ antenna rotation rate). For the low-elevation hazardous scan mode at $21.6^{\circ} \mathrm{s}^{-1}, n$ would be reduced to 14 .

The ground clutter spectrum is usually modeled as Gaussian with a squared width determined mainly by the antenna beam displacement (e.g., Doviak and Zrnić 1993):

$$
\sigma_{\mathrm{GC}}^{2}=\ln 2\left(\frac{\alpha \lambda \cos \phi}{2 \pi \theta_{\mathrm{BW}}}\right)^{2},
$$

where $\alpha$ is the antenna rotation rate, $\phi$ is the elevation angle ( $\sim 0$ for the lowest elevation scans), and $\theta_{\mathrm{BW}}$ $\left(0.55^{\circ}\right.$ for TDWR $)$ is the antenna beamwidth. For our example, we will assume a rotation rate of $19^{\circ} \mathrm{s}^{-1}$, which gives $\sigma_{\mathrm{GC}}=0.24 \mathrm{~m} \mathrm{~s}^{-1}$. However, ground clutter spectra can develop extended tails; for example, they can become exponential in form due to wind-blown vegetation (Billingsley 2002). Thus, we add a margin of $0.1 \mathrm{~m} \mathrm{~s}^{-1}$ to get $\sigma_{\mathrm{GC}}=\left[(0.24)^{2}+\left(0.1^{2}\right)\right]^{1 / 2}=0.26 \mathrm{~m} \mathrm{~s}^{-1}$. This is consistent with the FAA procedure for ground clutter specification. For the hazardous scan mode, the rotation rate would be faster, the clutter spectrum would be wider, and a different set of filters would need to be generated for a multi-PRI signal with smaller $n$.

The clutter rejection limit is set by transmitter stability. For the TDWR, the transmitter stability slightly exceeds the FAA's requirement of 55-dB clutter rejection, so we will design the maximum suppression filter to be $60 \mathrm{~dB}$. We will also generate filters at 40- and $20-\mathrm{dB}$ suppression levels for testing the suppression level selection algorithm.

\section{Filter design results}

Although the ideal output response specified in D should be the inverse of the clutter spectrum (which is usually assumed to be Gaussian), we found that the phase errors were significantly smaller in general if the ideal response was specified according to the traditional method of dividing the spectrum into three zones: stop band, transition band, and pass band. This choice is also justified by the fact that real ground clutter is often not Gaussian, as mentioned earlier. Phase error weights were set to zero in the stop band, while magnitude error weights were set to one in the pass band. Both types of error weights were set to zero in the transition band. The width of the transition band had subtle effects on the filter rolloff, and through trial and error we settled on a value of $0.2 \%$ of $v_{a \text { Min }}$, which is the unambiguous velocity corresponding to the longest PRI. For our case here, $v_{a \text { Min }}=14.1 \mathrm{~m} \mathrm{~s}^{-1}$. Experimentation also showed that, for our PRI sequences, acceptable phase responses were achieved using low values for the phase error weight in the pass band. Therefore, we designed the different suppression level filters by only varying 
the stop-bandwidth and stop-band magnitude error weight. The list of filter design parameters held fixed is given in Table 1.

Figure 1 shows the characteristics of the $60-\mathrm{dB}$ suppression clutter filter designed for the $4 \times 16$ MBS sequence. The frequency range was specified to cover the corresponding velocity range of $3 v_{a \mathrm{Min}}=42.3 \mathrm{~m} \mathrm{~s}^{-1}$, which satisfies the FAA velocity range requirement of $40 \mathrm{~m} \mathrm{~s}^{-1}$. The top panel in Fig. 1 shows the power suppression of the filter versus the normalized frequency. The transition between the stop and pass bands is rapid and smooth, and the response is reasonably flat even in the region where all of the PRIs are aliased (normalized frequency $>1.58$ ). The requested normalized stop bandwidth was 0.066 , while the actual normalized stop bandwidth was 0.064 as defined by the $3-\mathrm{dB}$ rise point from the minimum stop-band suppression, which was $59.1 \mathrm{~dB}$. The normalized pass-band edge, defined by the first point where the response reaches $-3 \mathrm{~dB}$, was 0.13 . The filter design parameters and actual characteristics are summarized in Table 2.

The middle panel of Fig. 1 shows the mean pulse-pair phase errors for the four PRI subsets. The values are normalized by $\pi$. Pulse-pair phase is the phase of the lag-1 autocorrelation of the signal, which is proportional to velocity. Its error is the difference in the mean pulse-pair phase computed for the input pulses and the output pulses. This quantity then yields a measure of the velocity bias introduced by the filter for each PRI subset. The response is quite linear in the pass band up to the frequency where all PRIs are aliased. Beyond this point, the errors still do not exceed about $6 \%$ of $\pi$ $\left(\sim 0.85 \mathrm{~m} \mathrm{~s}^{-1}\right)$.

For velocity dealiasing with the Chinese remainder theorem, it is imperative that the phase distortion not cause the velocity difference between PRI pairs to be identified with the incorrect quantized value; otherwise, false dealiasing results. For example, with a PRI ratio of $2: 3$, a velocity difference of zero corresponds to no aliasing, whereas a velocity difference of $v_{a \mathrm{Min}}$ corresponds to both velocities being aliased once. Therefore, if the velocity difference error exceeds $v_{a \mathrm{Min}} / 2$, then the dealiasing algorithm can either fail to dealias an aliased velocity or falsely dealias a nonaliased velocity. The velocity differences for the worst-case pulse-pair error $(\mathrm{PRI}$ ratio $=709: 945=3: 4)$ are shown in the bottom panel of Fig. 1. The dashed line indicates the theoretical velocity difference (for the case of no errors) used to

TABLE 1. Clutter filter design parameters kept constant.

\begin{tabular}{lc}
\hline \hline \multicolumn{1}{c}{ Parameter } & Value \\
\hline$M$ & 1025 \\
$N$ & 64 \\
Normalized transition bandwidth & 0.002 \\
Stop band phase weight & 0 \\
Pass band phase weight & 1 \\
Pass band magnitude weight & 1 \\
\hline
\end{tabular}
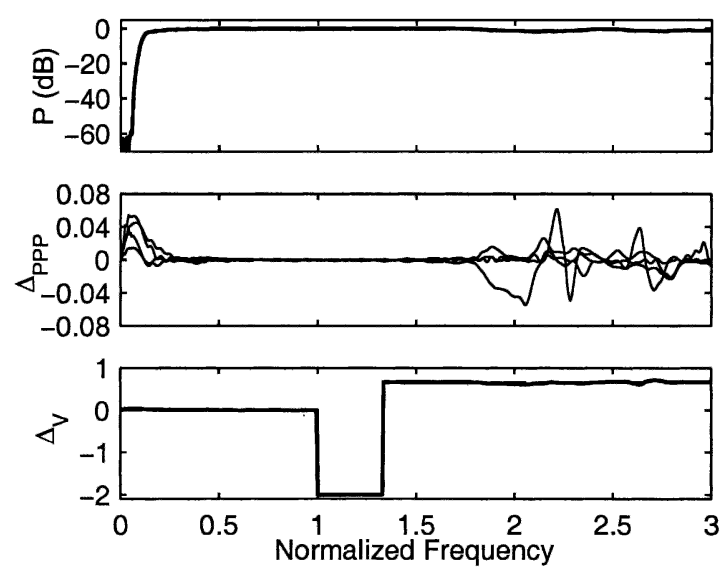

FIG. 1. (top) The power response for the $4 \times 16$ MBS clutter filter designed for $60-\mathrm{dB}$ suppression. Frequency is normalized by the unambiguous frequency corresponding to the longest PRI (945 $\mu$ s). (middle) The pulse-pair phase errors for the four PRI subsets. The errors are normalized by $\pi$. (bottom) The difference in velocity computed from PRI $=945 \mu$ s and PRI $=709 \mu$ s. The dashed line shows the theoretical case of no errors and the solid line shows the case where the pulse-pair errors are included. The velocity difference is normalized by the unambiguous velocity corresponding to PRI $=945 \mu \mathrm{s}\left(14.1 \mathrm{~m} \mathrm{~s}^{-1}\right)$.

unfold the measured velocity. The solid line shows the velocity difference with the pulse-pair phase error of the filter included. We see that the phase error will not cause false dealiasing, because the errors are much smaller than half the distance between the quantized steps.

As the suppression level is lowered, the phase response errors decrease as expected. Figure 2 shows the response for a $20-\mathrm{dB}$ filter. The maximum pulse-pair phase error is less than about $2 \%$ of $\pi$ in the pass band for all PRI subsets. Correspondingly, the errors in the velocity difference are also smaller. The key characteristics of the 40-dB filter are summarized in Table 2. The qualitative features of the magnitude and phase responses for this filter are similar to those shown in Figs. 1 and 2. The characteristics of MBS filters using all eight PRIs from our chosen set are also quite similar.

\section{Parameter estimation biases}

If part of the weather spectrum is removed by the clutter filter, then biases will be introduced in the estimated reflectivity $(Z)$, velocity $(V)$, and spectral width

TABLE 2 . The $4 \times 16$ MBS clutter filter design parameters and their actual characteristics.

\begin{tabular}{rcccc}
\hline \hline $\begin{array}{c}\text { Stop band } \\
\text { magnitude } \\
\text { weight }\end{array}$ & $\begin{array}{c}\text { Input stop } \\
\text { band edge } \\
\left(\mathrm{m} \mathrm{s}^{-1}\right)\end{array}$ & $\begin{array}{c}\text { Actual stop } \\
\text { band edge } \\
\left(\mathrm{m} \mathrm{s}^{-1}\right)\end{array}$ & $\begin{array}{c}\text { Pass band } \\
\text { edge } \\
\left(\mathrm{m} \mathrm{s}^{-1}\right)\end{array}$ & $\begin{array}{c}\text { Clutter } \\
\text { suppression } \\
(\mathrm{dB})\end{array}$ \\
\hline 500000 & 0.93 & 0.91 & 1.82 & 60.3 \\
8000 & 0.76 & 0.66 & 1.40 & 40.2 \\
60 & 0.63 & 0.50 & 0.99 & 20.8 \\
\hline
\end{tabular}



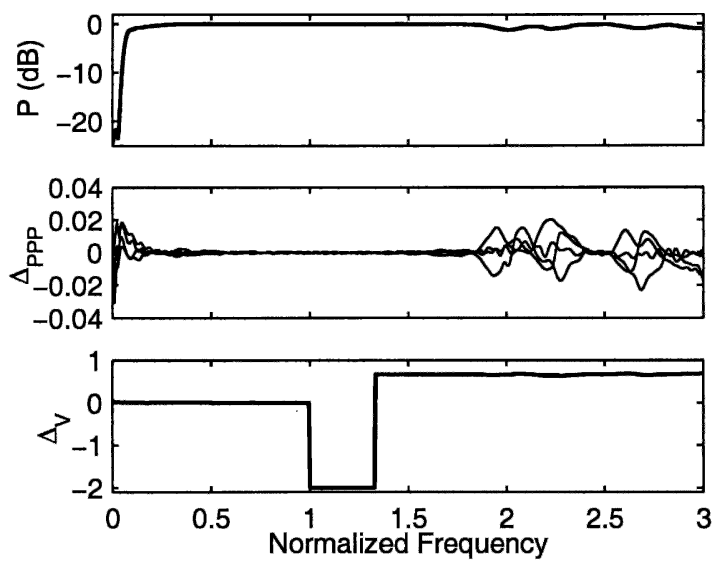

FIG. 2. (top) Power response, (middle) pulse-pair phase error, and (bottom) velocity difference for the $4 \times 16$ MBS clutter filter designed for $20-\mathrm{dB}$ suppression. See Fig. 1 caption for a more detailed description of the plots.

$\left(\sigma_{V}\right)$. For the TDWR, the FAA has the following clutter filter bias requirements at velocities equal to or greater than the minimum usable velocity: reflectivity bias $\leq 2 \mathrm{~dB}$, velocity bias $\leq 2 \mathrm{~m} \mathrm{~s}^{-1}$, and spectral width bias $\leq 2 \mathrm{~m} \mathrm{~s}^{-1}$. The minimum usable velocities are set to $2 \mathrm{~m} \mathrm{~s}^{-1}$ for $30-\mathrm{dB}$ filters and $4 \mathrm{~m} \mathrm{~s}^{-1}$ for $55-\mathrm{dB}$ filters. Other conditions under which these requirements must be met are signal-to-noise ratio $(\mathrm{SNR}) \geq 20 \mathrm{~dB}$, signalto-clutter residue ratio $\geq 30 \mathrm{~dB}$, and weather spectrum width $=1-4 \mathrm{~m} \mathrm{~s}^{-1}$.

To measure the filter bias we ran Monte Carlo simulations with synthesized weather plus clutter spectra sampled with the multi-PRI sequences, following the method of Zrnić (1975). For velocity estimation we used the standard pulse-pair method on each PRI subset, then took the median value across the subsets. For spectral width estimation we used lag 0 and lag 1 in the pulse-pair logarithm formula [Doviak and Zrnić 1993, their Eq. (6.27)]. Note that this formula has its own intrinsic bias as do other spectral width estimation methods. For each input parameter combination, 5000 simulation runs were made.

Figure 3 shows the reflectivity bias versus velocity for the $4 \times 16 \mathrm{MBS}$ clutter filters. The weather SNR was set to $20 \mathrm{~dB}$, while the clutter-to-noise ratio (CNR) was set to $10 \mathrm{~dB}$ below each filter suppression level. The FAA bias requirements are met for $V \geq 2 \mathrm{~m} \mathrm{~s}^{-1}(20-\mathrm{dB}$ filter), $V \geq 3 \mathrm{~m} \mathrm{~s}^{-1}$ (40-dB filter), and $V \geq 4 \mathrm{~m} \mathrm{~s}^{-1}$ (60-dB filter). These threshold velocities are comparable to the minimum usable velocities for the legacy clutter filters stated earlier. Figures 4 and 5 show the velocity and spectral width biases. For these parameters, the FAA bias requirements are met for all displayed velocities. As $v_{a \text { Min }}$ is approached and surpassed, aliasing effects introduce additional biases, but that is a separate issue that we will explore in Part II. Thus, overall, this set of filters meets the required bias specifications.

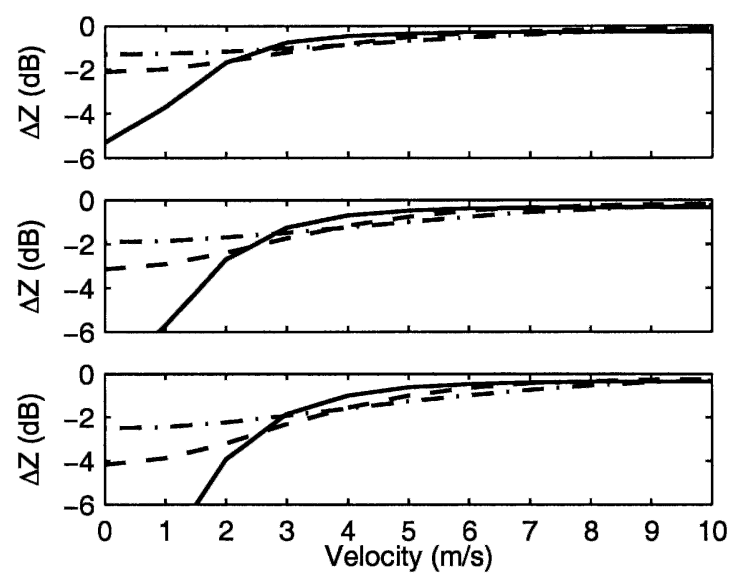

FIG. 3. Reflectivity bias vs weather velocity for the $4 \times 16$ MBS clutter filters. Filter suppression levels are (top) 20, (middle) 40, and (bottom) $60 \mathrm{~dB}$. The input CNRs were $10 \mathrm{~dB}$ below each filter suppression level. The input weather SNR was $20 \mathrm{~dB}$ with spectral widths of 1 (solid), 2.5 (dashed), and $4 \mathrm{~m} \mathrm{~s}^{-1}$ (dashed-dotted). The clutter spectral width was $0.26 \mathrm{~m} \mathrm{~s}^{-1}$. The input weather velocity points were spaced $1 \mathrm{~m} \mathrm{~s}^{-1}$ apart. A total of 5000 simulation runs were processed and averaged for each point.

\section{Adaptive filter selection}

Because the clutter filter-induced biases on the estimated parameters increase with suppression level, ideally the filter should be matched to the clutter level as closely as possible. One technique is to use a stored clutter residue map to estimate the signal-to-clutter ratio (SCR) after each filter has been applied, then the output from the lowest suppression filter that yields an acceptable SCR is used. This approach, however, cannot account for AP or the temporal variability of clutter. Thus, it is preferable to have a dynamic estimate of the clutter present in each cell.

We propose a method that utilizes clutter power estimated from an initial long-PRI scan that covers an
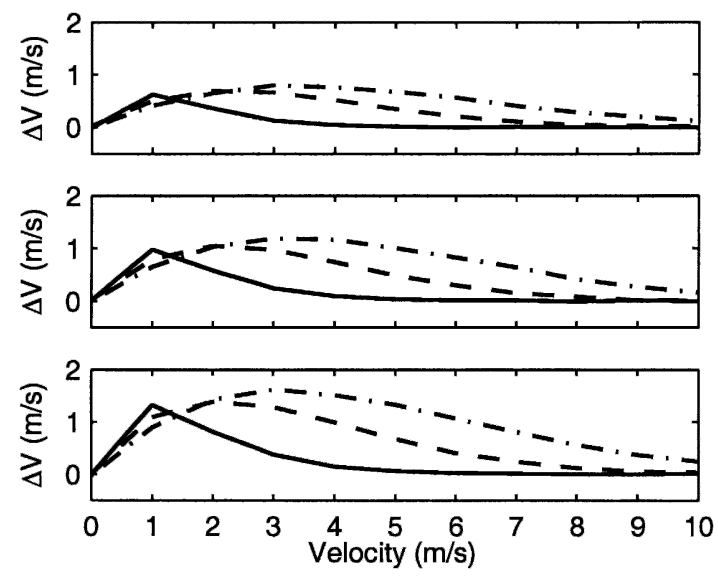

FIG. 4. Velocity bias vs weather velocity for the $4 \times 16$ MBS clutter filters. The simulation and filter parameters are the same as in Fig. 3. 

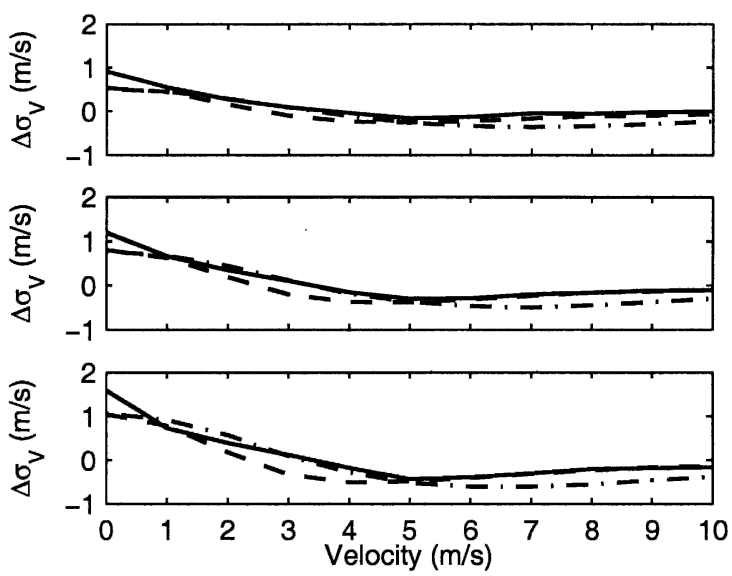

FIG. 5. Spectral width bias vs weather velocity for the $4 \times 16$ MBS clutter filters. The simulation and filter parameters are the same as in Fig. 3.

unambiguous range of $460 \mathrm{~km}$. (In Part II we will explain how this scan is also used for a range-fold protection scheme.) For this purpose we adopt the Gaussian model adaptive processing (GMAP) spectral domain clutter filtering technique (Siggia and Passarelli 2004). Based on the clutter spectral width due to antenna rotation and the power present in the spectrum near-zero Doppler, GMAP computes the theoretical Gaussian form of the clutter spectrum and removes the points for which this function is greater than the noise level. A Gaussian function is then generated using the computed spectral moments from the remaining points under the assumption that the clutter has been removed and only weather signals remain. The gap around zero Doppler is filled in using the spectral points of the Gaussian. The moments are recomputed and the gap refilled until there is reasonable convergence. (Clearly, it is assumed that the weather spectrum can be adequately represented by a single Gaussian.) The aim of GMAP is to reduce the clutter filter bias by filling in the stop band with spectral points that are modeled to follow the remaining weather spectrum. GMAP is being considered for use in the upcoming WSR-88D Open Radar Data Acquisition (ORDA) system, and testing has shown it to meet all clutter filter requirements (Ice et al. 2004).

In our case, the purpose of applying the GMAP clutter filter to an initial long-PRI scan is to estimate the clutter power present. The idea is that even if part of a weather spectrum impinging on the presumed clutter zone around zero Doppler is removed, GMAP will refill the clutter gap properly and restore the removed weather signal. Thus, the estimate of clutter power should be better than if all of the removed power was assumed to be clutter.

To test the effectiveness of GMAP on a long-PRI scan for clutter estimation, we ran Monte Carlo simulations as described earlier, except with the weather and clutter spectra sampled by a constant PRI of 3066 $\mu \mathrm{s}$. The number of pulse samples per run was 15 (to match the monitor scan dwell time). The clutter power was estimated as the difference in power between the unfiltered signal and the GMAP-filtered signal. If the filtered power was greater than the input power, the clutter power was assigned to zero.

For the case of no weather signal present, the clutter estimation bias was essentially negligible. With weather signals of spectral width $1-4 \mathrm{~m} \mathrm{~s}^{-1}$, clutter estimation bias was also negligible for clutter-to-signal ratio (CSR) greater than about $10 \mathrm{~dB}$. The only condition under which the bias became large was for small CSRs with narrow weather spectra. This is illustrated in Fig. 6. Clearly, as the weather spectrum narrows and its velocity approaches zero, it becomes impossible to distinguish it from clutter. Still, the maximum bias did not exceed $2.4 \mathrm{~dB}$ at CSR $=0 \mathrm{~dB}$. For our long-PRI signal, because the unambiguous velocity is only $4.3 \mathrm{~m} \mathrm{~s}^{-1}$, the weather spectrum wraps around to zero as the velocity is increased further, correspondingly raising the bias again. Overall, the clutter power estimation performance of GMAP is excellent and provides a solid basis for choosing the best suppression level for the multiPRI clutter filter.

In the next stage of the adaptive clutter filter selection scheme, we first compute the zero Doppler power component in the multi-PRI signal. If this power is greater than the noise power, then clutter filtering is considered. Otherwise, no clutter filter is applied. This test is mainly aimed at cases (as seen in Fig. 6) where aliased weather spectra in the long-PRI scan cause false clutter detection by GMAP.

Next, the clutter filter level is selected according to

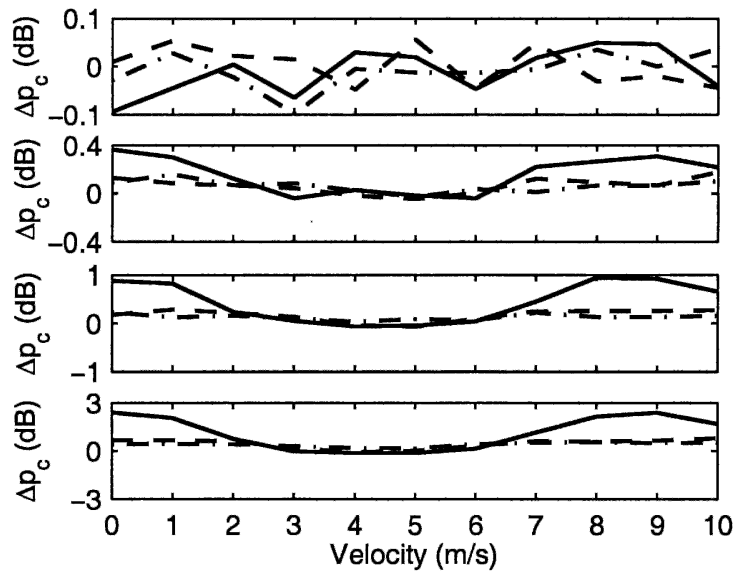

FIG. 6. GMAP clutter power estimation bias vs weather velocity for the case of (top to bottom panels) CSR $=15,10,5$, and $0 \mathrm{~dB}$. The weather spectral widths were 1 (solid), 2.5 (dashed), and 4 $\mathrm{m} \mathrm{s}^{-1}$ (dashed-dotted). The weather SNR was $20 \mathrm{~dB}$. Fifteenpoint digital Fourier transforms (DFTs) were used in the spectral calculations, with PRI $=3066 \mu$ s. The input weather velocity points were spaced $1 \mathrm{~m} \mathrm{~s}^{-1}$ apart. A total of 5000 simulation runs were processed and averaged for each point. 
the estimated CNR. For CNR $<0 \mathrm{~dB}$, no clutter filter is applied. For $0 \mathrm{~dB} \leq \mathrm{CNR}<20 \mathrm{~dB}$, the 20 -dB filter is applied. For $20 \mathrm{~dB} \leq \mathrm{CNR}<40 \mathrm{~dB}$, the 40 - $\mathrm{dB}$ filter is applied. For $\mathrm{CNR} \geq 40 \mathrm{~dB}$, the 60 -dB filter is applied. If a finer gradation of the suppression levels is desired, this can easily be changed, for example, to $10-\mathrm{dB}$ increments instead of $20 \mathrm{~dB}$.

Finally we present the parameter estimation biases resulting from this adaptive filter selection scheme. As before, simulated clutter and weather signals were generated, first, for constant, long-PRI pulses on which GMAP was applied to estimate the clutter power, then for the $4 \times 16 \mathrm{MBS}$ signal on which the selected level of clutter filter was applied according to the GMAPestimated clutter power. The reflectivity bias results are shown in Fig. 7. For a $20-\mathrm{dB}$ weather signal, the top panel in Fig. 7 shows the reflectivity bias with no clutter present. The only significant bias is for a weather spectral width of $1 \mathrm{~m} \mathrm{~s}^{-1}$. At velocities close to zero, the narrow weather spectrum appears too much like clutter and a fraction of the power is removed. This is basically the drawback of having an adaptive clutter filter scheme. It seems, however, a small price to pay for gaining the ability to filter AP signals and to dynamically adapt to changing ground clutter conditions. Note that the reflectivity biases for $\mathrm{CNR}=10,30$, and $50 \mathrm{~dB}$ are as good as the corresponding cases in Fig. 3 where the same CNRs were filtered by 20-, 40-, and 60-dB filters. In fact, the bias is even smaller with the adaptive filter for the CNR $=10 \mathrm{~dB}$ case. This is because the selection algorithm picks not only the $20-\mathrm{dB}$ filter, but also sometimes no filter, so the biases in opposite directions tend to cancel out.

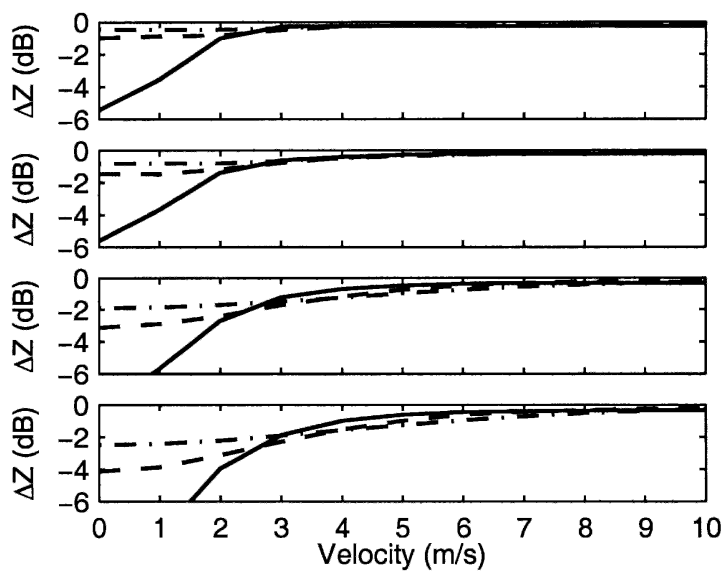

FIG. 7. Reflectivity bias vs weather velocity for adaptively selected $4 \times 16$ MBS clutter filters. The top panel shows the case with no input clutter power. The next three panels show cases with input CNR of 10, 30, and $50 \mathrm{~dB}$. The input weather SNR was 20 $\mathrm{dB}$ with spectral widths of 1 (solid), 2.5 (dashed), and $4 \mathrm{~m} \mathrm{~s}^{-1}$ (dashed-dotted). The clutter spectral width was $0.26 \mathrm{~m} \mathrm{~s}^{-1}$. The input weather velocity points were spaced $1 \mathrm{~m} \mathrm{~s}^{-1}$ apart. A total of 5000 simulation runs were processed and averaged for each point.
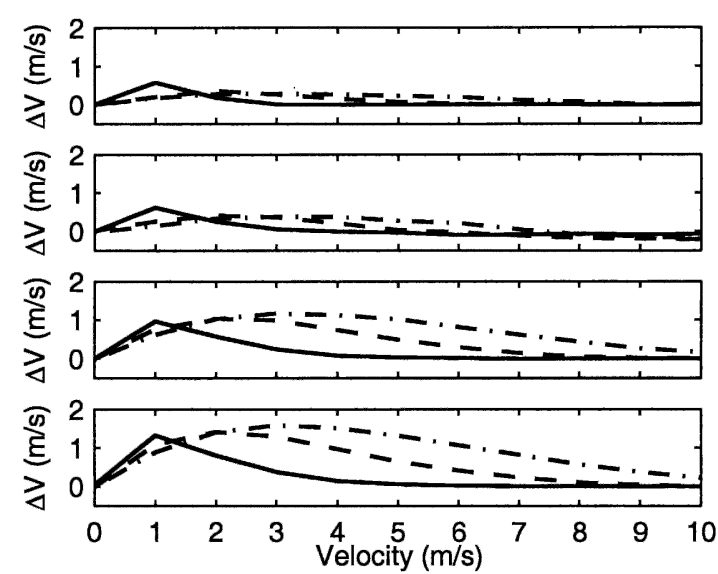

FIG. 8. Velocity bias vs weather velocity for adaptively selected $4 \times 16$ MBS clutter filters. The simulation and filter parameters are the same as in Fig. 7.

In Fig. 8, the velocity bias results are shown. The no-clutter case indicates small amounts of bias, but they are much less than the FAA limit of $2 \mathrm{~m} \mathrm{~s}^{-1}$. Again, in comparison to the fixed-level filter results of Fig. 4, the biases compare favorably. As noted above, the occasional selection of no filtering for the CNR $=10 \mathrm{~dB}$ case results in a compensatory bias, which shows up as a slightly negative bias at larger velocities. Similar comments apply to the spectral width bias results shown in Fig. 9.

We also repeated the above simulation tests with $\mathrm{CNR}=20$ and $40 \mathrm{~dB}$. In these cases, the CNRs were right on the edge of the quantized suppression levels, so one might expect some increase in estimation bias due to inadvertent oversuppression. Indeed, there were increases in the biases, but they were quite small and the overall results were still well within FAA limits.

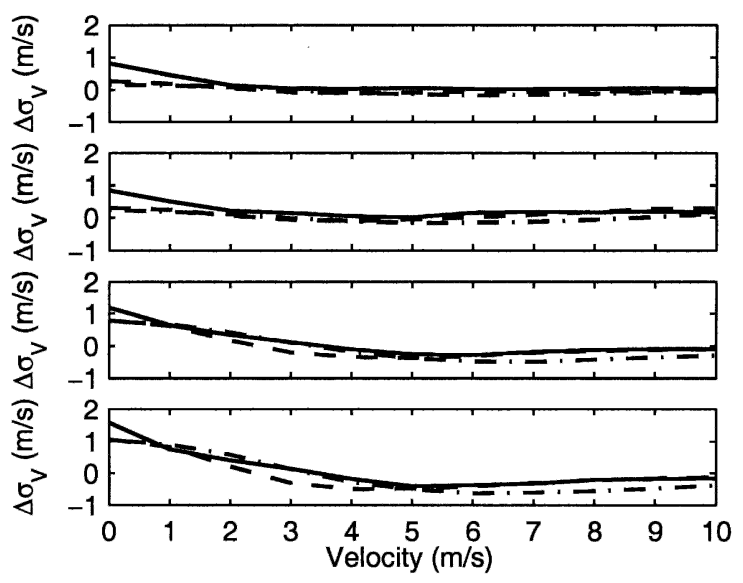

FIG. 9. Spectral width bias vs weather velocity for adaptively selected $4 \times 16$ MBS clutter filters. The simulation and filter parameters are the same as in Fig. 7. 


\section{Discussion}

Although we have only shown results for a $4 \times 16$ MBS PRI sequence, we are currently planning to also use an $8 \times 8$ MBS pulse train for the monitor scan mode. The additional diversity in PRI sets provides more flexibility in range-fold protection. Of course, it is possible to have available many more sets of multi-PRI sequences from which to select. It is a matter of balancing the payoff against the mounting complexity and required memory. From a clutter filtering perspective it is not a problem, because simulation studies such as presented in this paper have shown filters for other MBS combinations to perform just as well as for the 4 $\times 16$ filters.

The clutter filter technique presented in this paper is certainly applicable to radars besides the TDWR. For example, staggered PRI sequences are being considered for use in the new WSR-88D ORDA. If the sequences are rearranged as block-staggered patterns, our FIR filter design program can produce very effective filters. Since the WSR-88D ORDA also plans to utilize an initial long-PRI scan at low elevation angles, our adaptive filter selection approach can be implemented.

The GMAP clutter filter, which we use to estimate the clutter power present, takes as input the presumed Gaussian clutter spectral width. However, real ground clutter spectra are not necessarily Gaussian. We, therefore, added a feature to search the spectrum outward, starting from the points where the presumed Gaussian falls to the noise level, for upward inflection points. The purpose is to extend the clutter window, if necessary, to follow a non-Gaussian tail. We limited this search to two points, however, in case a weather spectrum tail appears like a clutter spectrum tail. This can be adjusted based on more extensive studies using real data. All the results in this paper included this modification to the GMAP algorithm, even though the simulated spectra were all Gaussian, because this is the algorithm that we apply to real data.

\section{Conclusions}

We showed in this paper that FIR clutter filters with excellent magnitude and phase responses can be designed for MBS signals. Biases in reflectivity, velocity, and spectral width resulting from these filters were demonstrated to satisfy requirements equivalent to the FAA's specifications for the current TDWR clutter filters. We then proposed an adaptive scheme in which estimates of clutter power from an initial long-PRI scan are used to select the filter with the appropriate clutter suppression level. The GMAP clutter filter was employed to estimate the long-PRI clutter power, and we showed that the estimate errors were very low for CSR $\geq 10 \mathrm{~dB}$. Finally, we ran simulations to quantify the biases resulting from this adaptive clutter filter algo- rithm. The results were excellent and provide confidence that this clutter filter scheme will work well for multi-PRI signals in the enhanced TDWR signal processing system.

In Part II of this series of papers we will present, and discuss the results of, algorithms used on the received multi-PRI signals for range-fold protection and velocity dealiasing.

Acknowledgments. This work was sponsored by the Federal Aviation Administration under Air Force Contract F19628-00-C-0002. Opinions, interpretations, conclusions, and recommendations are those of the authors and are not necessarily endorsed by the U.S. government.

\section{REFERENCES}

Banjanin, Z. B., and D. S. Zrnić, 1991: Clutter rejection for Doppler weather radars which use staggered pulses. IEEE Trans. Geosci. Remote Sens., 29, 610-620.

Billingsley, J. B., 2002: Low-Angle Radar Land Clutter: Measurements and Empirical Models. William Andrews, 722 pp.

Cho, J. Y. N., 2003: Evaluation of TDWR range-ambiguity mitigation techniques. Project Rep. ATC-310, MIT Lincoln Laboratory, Lexington, MA, $47 \mathrm{pp}$.

_, G. R. Elkin, and N. G. Parker, 2003: Range-velocity ambiguity mitigation schemes for the enhanced terminal Doppler weather radar. Preprints, 31st Conf. on Radar Meteorology, Seattle, WA, Amer. Meteor. Soc., 463-466.

Chornoboy, E. S., 1993: Clutter filter design for multiple-PRT signals. Preprints, 26th Conf. on Radar Meteorology, Norman, OK, Amer. Meteor. Soc., 235-237.

Doviak, R. J., and D. S. Zrnić, 1993: Doppler Radar and Weather Observations. Academic Press, 562 pp.

Fang, M., R. J. Doviak, and V. Melnikov, 2004: Spectrum width measured by WSR-88D: Error sources and statistics of various weather phenomena. J. Atmos. Oceanic Technol., 21, 888-904.

Ice, R. L., R. D. Rhoton, D. S. Saxion, N. K. Patel, D. Sirmans, D. A. Warde, D. L. Rachel, and R. G. Fehlen, 2004: Radar Operations Center (ROC) evaluation of the WSR-88D Open Radar Data Acquisition (ORDA) system signal processing. Preprints, 20th Int. Conf. on Interactive Information and Processing Systems for Meteorology, Oceanography, and Hydrology, Seattle, WA, Amer. Meteor. Soc., 5.5.

Sachidananda, M., and D. S. Zrnić, 1999: Systematic phase codes for resolving range overlaid signals in a Doppler weather radar. J. Atmos. Oceanic Technol., 16, 1351-1363.

, and _ 2000: Clutter filtering and spectral moment estimation for Doppler weather radars using staggered pulse repetition time (PRT). J. Atmos. Oceanic Technol., 17, 323-331.

Siggia, A., 1983: Processing phase coded radar signals with adaptive digital filters. Preprints, 21st Int. Conf. on Radar Meteorology, Edmonton, AB, Canada, Amer. Meteor. Soc., 167172.

_ and R. E. Passarelli Jr., 2004: Gaussian model adaptive processing (GMAP) for improved ground clutter cancelation and moment estimation. Preprints, Third European Conf. on Radar in Meteorology and Hydrology, Visby, Sweden, Copernicus Gesellschaft, 67-73.

Weber, M. E., 2002: ASR-9 Weather Systems Processor (WSP) signal processing algorithms. Project Rep. ATC-255, MIT Lincoln Laboratory, Lexington, MA, $53 \mathrm{pp}$.

Zrnić, D. S., 1975: Simulation of weatherlike Doppler spectra and signals. J. Appl. Meteor., 14, 619-620. 